\title{
Las reformas del palacio de los marqueses de Valdecarzana en Oviedo
}

\author{
Juan Díaz Álvarez \\ Universidad de Oviedo
}

RESUMEN:

La casa de Miranda fue, junto con la de los Quirós, una de las principales de Asturias, alrededor de las que se polarizó la vida política en la región, como cabezas de bando. Sendas familias tenían residencia en Oviedo. Este artículo trata sobre las reformas barrocas acometidas por los Miranda, marqueses de Valdecarzana, en su palacio ovetense, adecuándose a la ordenación de la ciudad asturiana en diferentes épocas, así como a las nuevas estéticas.

PALABRAS CLAVE:

Gonzalo de Güemes, Gregorio de la Roza, Manuel Reguera, Marqueses de Valdecarzana, Casa de Heredia, Oviedo, Barroco, Ilustración

\section{ABSTRACT:}

Miranda's and Quirós' families were principal in Asturias during Ancien Régime, since late middle age. They polarized the politic in the region. Paths families had homes in Oviedo. This paper is about the construction process of Miranda's house-palace during Ancien Regime, adapting to the management of the asturian city at different times, as well as new aesthetic.

\section{KEY WORDS:}

Gonzalo de Güemes, Gregorio de la Roza, Manuel Reguera, Marquises of Valdecarzana, Heredia Family, Oviedo, Baroque, Enlightenment 


\section{Introducción}

El actual palacio de Valdecarzana, sito en la ovetense calle de San Juan, es el resultado de un proceso constructivo que se alarga desde la primera mitad del siglo xvi a la segunda del xVIII. La primera noticia que tenemos de este inmueble la facilita un inventario de bienes realizado tras el fallecimiento de Sancho de Miranda, titular de la casa y mayorazgo de la misma denominación y pariente mayor del Principado, y su esposa, Leonor de las Alas, hacia 1550. En ese documento se citan una serie de bienes que habían sido adquiridos por el matrimonio y agregados al vínculo, entre ellos "las casas de Oviedo" valoradas en 375.000 maravedíes (mil ducados de oro) ${ }^{1}$. La segunda de aquellas fechas alude a la última intervención sobre el complejo constructivo, la encargada en un principio a Manuel Reguera, por la familia Heredia, que había aforado la vieja casa de los Miranda, marqueses de Valdecarzana, ampliándola a costa del solar que ocupaba la residencia de los Hevia, miembros de la oligarquía ovetense.

En un estudio pionero, el profesor Ramallo Asensio, al tipificar la arquitectura civil asturiana levantada a lo largo de la Edad Moderna, y más en concreto la residencial, databa este edificio en la segunda mitad del siglo $\mathrm{XVII}^{2}$, si bien más tarde, el profesor Vidal de la Madrid, consideraba acertado retrasar unas décadas la edificación, basándose en la novedad de su traza en el contexto urbano de la ciudad, apuntando como fecha más propicia los últimos años de la década de 1620. Ambas posturas son acertadas y se sustentan en sendos procesos de reforma del inmueble a lo largo de la centuria. Una en 1628 debida a la mano del maestro Gonzalo de Güemes Bracamonte y la iniciativa del santiaguista y regidor don Diego de Miranda; la otra en 1668, protagonizada por el recién llegado arquitecto Gregorio de la Roza, bajo el patrocinio del segundo marqués de Valdecarzana, don Lope Fernández de Miranda Ponce de León ${ }^{3}$. Junto a estos maestros hay que tener presente al candasín Manuel Reguera González, que se encargaría de la última de las reformas del edificio, que le da el aspecto actual, a petición de los Heredia, en la década de $1770^{4}$.

\section{Arquitectura y vanguardia en el palacio Valdecarzana}

De estas intervenciones puede inferirse la vinculación de este conjunto residencial a la vanguardia estilística de la época. Gonzalo de Güemes ( + c. 1639), junto con Pedro de Cubas de la Huerta y Juan de Naveda (1590-1638), fueron los responsables de la introducción de la estética post-herreriana en el Principado, dando lugar a un primer barroco muy tímido en sus arrebatos decorativos, siempre muy contenidos, de herencia clasicista. Si bien es cierto que la historiografía viene en reafirmar en todo este proceso la importancia de Naveda, lo cierto es que, antes que él, en Asturias estaban trabajando los anteriores, el primero desde su posición para el consistorio ovetense de fontanero de la ciudad, el segundo, sobre todo en Gijón, donde ya había proyectado la capilla del palacio de los Valdés. Quizás la relevancia del tercero se deba a que da el empujón definitivo a este nuevo concepto estilístico y su importancia en el panorama constructivo asturiano se debió al hecho de proceder del conjunto residencial patrocinado por el duque de Lerma en la villa burgalesa del mismo nombre, hacerse con el contrato catedralicio para finalizar las obras del templo, su girola, y ser nombrado, por ende, maestro mayor de obras de la catedral. Su posición de preeminencia le hizo que fuera buscado por otros importantes promotores urbanos, uno lo fue la Justicia y Regimiento de la ciudad, quien le encargó las trazas para un nuevo edificio consistorial, otro lo fue la misma oligarquía urbana, que deseaba reformar sus viejas residencias y adaptarlas

Abreviaturas: ACo (Archivo de la Catedral de Oviedo), AHA (Archivo Histórico de Asturias), AMG (Archivo Municipal de Gijón), AMO (Archivo Municipal de Oviedo), ARIDEA (Archivo del Real Instituto de Estudios Asturianos), FM (Fondo Marcenado), PG (Protocolos de Gijón), PO (Protocolos de Oviedo).

ARIDEA, Casa de Miranda, caja 2, doc. 3: c. 1550, s/f.

Ramallo Asensio, G.: La arquitectura civil asturiana (Época Moderna), Salinas, Ayalga, 1978, p. 124.

\footnotetext{
AHA, PO, cajas 7066 (Oviedo, 29-XI-1628, s/f) y 7319 (Oviedo, 4-v-1668, ff. 39r-42r), apud KaWAmURA, Y.: Arquitectura y poderes civiles. Oviedo, 1600-1680, Oviedo, Ridea, 2006, pp. 157 (n. 541) y s. (n. 546), respectivamente.

4 Sobre la intervención de Reguera vid. Madrid Álvarez, V. de la: La arquitectura de la Ilustración en Asturias. Manuel Reguera, 1731-1798, Oviedo, RIDEA, 1995, pp. 142 y ss.
} 
a los nuevos parámetros estéticos, de habitabilidad y de domesticidad ${ }^{5}$.

Entonces, ¿cómo don Diego de Miranda, miembro destacado del regimiento ovetense, se decantó por la figura de Güemes en vez de la nueva estrella llegada, Naveda? La documentación no permite dar una respuesta tajante, pero téngase en cuenta, que el mismo Cabildo catedralicio, solicitó un proyecto a Güemes para que se encargara del remate del templo en la temprana fecha de 1617, si bien el asunto quedó latente hasta que la institución eclesiástica se decidió por la traza de Naveda unos años más tarde, quizás por influencia de su hermano, el canónigo-doctoral don Francisco de Naveda. El primero estaba plenamente asentado en la vida constructiva asturiana, a la que había llegado en las postrimerías del siglo xvi, de la mano de su suegro Pedro de la Bárzana, procedente de Monforte de Lemos donde trabajó en el colegio jesuítico junto a Diego Vélez. La primera referencia documental que se tiene del maestro Güemes en el Principado data de 1599 a raíz de su intervención en el claustro del convento franciscano de Avilés. Su trabajo en la región se estabiliza al ser nombrado maestro de obras y fontanero del consistorio ovetense en 1611, encargándose de las obras edilicias ${ }^{6}$, entre las que sobresalió la fuente de Fitoria. La importancia de esta obra se debe a que era el principal abasto de agua para la ciudad. El fontanero debía encargarse de que no faltara el suministro, así como del mantenimiento de las principales fuentes del entramado urbano y el sistema de cañerías ${ }^{7}$. La reputación de este trasmerano fue aumentando: tanteado por el cabildo catedralicio para que se hiciera cargo de la

\footnotetext{
Sobre la trascendencia de la obra Naveda vid. Losada VAREA, C., La arquitectura en el otoño del Renacimiento. Juan de Naveda (1590-1638), Santander, Universidad de Cantabria, 2007. Una visión ajustada de su producción arquitectónica en Asturias en Díaz Álvarez, J., «La obra del arquitecto Juan de Naveda o la recepción del clasicismo herreriano en la Asturias del primer tercio del siglo xviIn, en I Congreso de estudios asturianos. Tomo V. Comisión de Artes, arquitectura y urbanismo, Oviedo, RIDEA, 2007, pp. 31-50.

6 Pastor Criado, Ma I.: La arquitectura purista en Asturias, Oviedo, Consejería de Cultura, 1987, p. 189.

7 Sobre estas cuestiones vid. PASTOR CRIADO, M. ${ }^{a}$ I., La arquitectura purista..., pp. 100 y ss. y «El acueducto de los Pilares de Oviedon, Liño, 7, 1987, pp. 39-54; Fernández Álvarez, J. M., La fuente de Fitoria: el problema de abastecimiento de agua a la ciudad de Oviedo (15681613), Oviedo, Universidad de Oviedo, 1996; KawamuRA, Y., Arquitectura y poderes civiles..., pp. 47-76.
}

girola de la catedral, trabajó en la iglesia parroquial de San Félix de Candás, en el puerto y cay de Lastres en $1624^{8}$, intervino en la capilla que los Merás promocionaron en el convento franciscano de Tineo en 1629, si bien cedió la obra, que había heredado de su cuñado ${ }^{9}$ al final de sus días fue contratado por la cofradía gijonesa de Nuestra Señora de Contrueces, a través de su mayordomo, a la sazón, Alonso Ramírez de Jove, regidor de Oviedo y de Gijón, para la realización de unas trazas orientadas a reformar de la capilla mayor del santuario mariano, en 1638, aunque las obras fueron materializadas por Francisco de Cubas y Simón Tío ${ }^{10}$.

Por su parte, Gregorio de la Roza (Carriazo, c. 1643-0viedo, 1706), recién llegado a Oviedo, se vincula a importantes proyectos municipales junto a los maestros Juan de Estrada y Pablo de Cubas Ceballos, como la casa de los corregidores en la calle de la Rúa y las nuevas carnicerías; accede más tarde al taller del destacado maestro de la catedral, Ignacio del Cajigal, uno de los principales introductores el pleno barroco, basado en la riqueza decorativa, y tras su óbito se casó con su viuda, Francisca de Palacio, en 1669, haciéndose, por ende, con su clientela. La relación de Roza con los representantes del poder municipal, a pesar de su juventud, por su intervención en obras edilicias, y su pertenencia al equipo de Cajigal, pudieron inclinar al marqués don Lope a contratar sus servicios para la reforma de su casa ovetense. En el mismo año de su matrimonio con Francisca, de la Roza se hace con otro importante contrato, la casa de los Malleza en la Plaza de la Fortaleza ${ }^{11}$ y, a mediados de la década de 1670 , la promo-

$8 \quad$ AHA, PO, caja 6938: Oviedo, año 1624, s/f.

9 AHA, P0, caja 7045: Oviedo, 23-vI-1629, s/f y Oviedo, 25-VI-1629, s/f

10 AHA, PG, caja 1796-1: Gijón, 22-II-1638, s/f.

11 La historiografía venía en considerar que la casa de los Malleza había sido contratada en la década de 1670; investigaciones de la profesora Kawamura retrasan esa fecha a las postrimerías de la década anterior, al documentar el boceto de las trazas y el contrato de obras a finales de 1669 (KaWAmURA, Y.: "Precisiones sobre la construcción de la casa palacio de Fernando de Malleza y Doriga en Oviedo», BRIDEA, 161, 2003, p. 163). Los promotores de este edificio fueron, doña Isabel de Malleza y Miranda, titular del vínculo de Malleza, y su esposo el regidor ovetense y santiaguista don Fernando de Malleza y Doriga, mayorazgo de Doriga. En realidad fue las obras suponen la reforma de la antigua residencia en Oviedo de los Malleza. La elección del maestro de la Roza pudo estar condicionada por la que en su momento hizo el marqués de Valdecarzana, don Lope, primo carnal de doña Isabel, pero no ha de obviarse la 
vida por el calatravo don Sebastián Vigil de la Rúa, vecina a la anterior ${ }^{12}$. En ambos casos, los promotores eran miembros destacados del consistorio de la ciudad. La estrella del trasmerano fue ascendiendo y nombrado maestro de obras del Principado a petición de la Junta General en 1672.

La principal aportación de Manuel Reguera González (Candás, 1731-Oviedo, 1798) al oficio constructivo en Asturias es su adecuación a los nuevos parámetros ilustrados implantados en época de Carlos III; formado en la tradición arquitectónica bajo el magisterio de Pedro Antonio Menéndez, maestro a medio camino entre el Barroco y la Ilustración, hubo de viajar a Madrid para obtener el título de arquitecto que otorgaba la Real Academia de Bellas Artes de San Fernando. Una vez logrado, entabló batalla contra aquéllos que en Asturias no habían pasado tal trámite, el principal de ellos José Bernardo de la Meana, su opositor más fuerte y cualificado. En el fondo, lo que estaba en juego era la preeminencia en el oficio y la obtención de los contratos más suculentos, procedentes de la Iglesia y de las instituciones locales $\mathrm{y}$ regionales y de los de promoción regia en el Principado. La Corona, con la expedición de los títulos de arquitectura por su Academia, se aseguraba la introducción del academicismo en el reino con la finalidad de desplazar los presupuestos barrocos, asociados al casticismo de la dinastía precedente en el trono: los Austrias. La titulación por la Academia y la obtención de contratos de promoción del Estado favoreció la formación de unas redes de relaciones entre la Corte y la periferia, haciendo frente a los grupos sociales más reaccionarios al programa de reformas de la monarquía ilustrada carolina en materia artística, sobre todo el estamento eclesiástico. Esta relación entre Asturias y la institución madrileña de Bellas Artes se entabla a través de Reguera, como el primer arquitecto asturiano titulado y miembro de mérito de San Fernando; Ventura Rodríguez, principal artífice de algunos de los proyectos de la Monarquía a lo largo del reino; y uno de los principales representantes de la Ilustración, oriundo de la región, don Gaspar Melchor de Jovellanos.

vinculación del arquitecto con diversos miembros de la oligarquía urbana.

12 AHA, P0, caja 7665: 0viedo, 9-XII-1675, ff. 5r-6r (apud Madrid Álvarez, V. de la: "El arquitecto Juan de Estrada y la persistencia del clasicismo en Asturias», BSAA. Arte, 79, 2013, p. 105).
La proyección que alcanzaría el candasín fue intuida por varios miembros de la oligarquía urbana, que solicitaron sus servicios para que reformara o construyera sus moradas de residencia, tales como los Velarde, los Inclán, los Heredia o los Bernaldo de Quirós Benavides; también trabajó para la Junta General en el ámbito de la obra civil (en la carretera de Castilla o en puerto de Gijón), para el ayuntamiento ovetense en obras edilicias como el empedrado de calles y reformas de fuentes en su calidad de fontanero de la ciudad; la reforma de algunos templos parroquiales como en El Pino (Aller), en Cenero (Gijón) o en el frontis de la iglesia conventual de los dominicos en Oviedo. ${ }^{13}$

\section{Los procesos constructivos de las reformas}

Los Miranda, una de las familias preeminentes en la Asturias del Antiguo Régimen, junto con los Quirós, polarizaron la vida política asturiana en tanto que fueron cabeza de bando desde la Baja Edad Media. Ambas disponían de residencia en Oviedo; los Quirós, una casa-torre en la calle de Platería, los Miranda en la calle de San Juan. Estas construcciones hay que llevarlas, al menos a mediados del siglo xv; la de los Quirós se cita en la escritura de su vínculo de 1474 y la de los Miranda, como mencioné, solo se rastrea desde la primera mitad del siglo xvi. Juan Uría Ríu no la menciona en su artículo sobre las torres urbanas de Oviedo, por lo que hay que suponer que fuera una construcción más modesta, a pesar de que en la actualidad conserva una torre en la esquina $\mathrm{N}-\mathrm{O}^{14}$, cuya fábrica sea con probabilidad posterior. El edificio sufre dos importantes procesos de reforma a lo largo del siglo xvil, y las autorías corren a cargo de Gonzalo de Güemes y de Gregorio de la Roza, como ya avancé. Aunque no he podido localizar el contrato sobre la primera, la personalidad de Güemes queda reflejada indirectamente, al ser mencionado "maestro de la obran a través de un contrato con el cante-

\footnotetext{
13 Sobre la obra de Reguera vid. Llaguno y Amírola, E. de y J. A. CeÁn Bermúdez: Noticias de los arquitectos y arquitectura de España desde su restauración, ilustradas y acrecentadas con notas, adiciones y documentos, t. IV, Madrid, Imprenta Real, 1829, pp. 319-321; MADRID Álvarez, V. de la: La arquitectura de la Ilustración....

14 URÍ Ríu, J., "Contribución a la historia de la arquitectura regional: las casas de 0viedo en la diplomática de los siglos XIII al XVI", BIDEA, 60, 1967, 3-30.
} 
ro Francisco González de Horna, encargado de subministrar piedra a la obra ${ }^{15}$.

La mención a Güemes es reveladora, pero la carencia del documento directo que lo vincula a la promoción de los Miranda hace sobrevolar cierta duda sobre el proyecto, ¿fue este «maestro" el encargado de hacer las trazas y condiciones en las que se desarrollarían la obra?, ¿o fue un mero aparejador, supeditado al diseño de otro profesional más cualificado? De ser afirmativo el primer interrogante, su figura cobra mayor importancia en el contexto constructivo asturiano, y sobre todo en el ovetense, como capital de la región y principal concentrador de promotores en la época, sobre todo la Iglesia. En favor de esta hipótesis hay que recordar cómo el cabildo le solicitó un proyecto para la girola de la catedral, que debía de tenerlo en estima como tracista para encargarle esta tarea tan delicada, aunque quedaría supeditado a la preeminencia de Naveda en la Asturias del segundo cuarto del siglo xvII, que se hizo con el contrato definitivo, avalado por su fama como proyectista, amén de su parentesco con uno de los canónigos ${ }^{16}$.

El palacio estaba localizado en un solar frontero por el Norte con la iglesia y hospital real de San Juan, separados por una estrecha calleja con la misma denominación (en la actualidad calle de Schultz). Con la progresiva llegada de la nobleza regional a la ciudad y su pretensión de establecimiento intramuros, los solares bien situados escaseaban produciéndose un abigarramiento de las construcciones; los problemas de espacio se solucionan con intromisiones de unas construcciones sobre las otras produciendo continuos conflictos y pleitos. Contenciosos de este tipo fueron habituales durante la Edad Moderna, los que surgen en un contexto de escasez de suelo urbanizable y de una trama viaria irregular de herencia medieval. La primera reforma barroca del edificio la conocemos por uno de estos sucesos, que tuvo lugar con el citado hospital «E dixeron que por quanto el dicho señor Don Diego de Miranda rehedificava

15 AHA, P0, caja 7066: Oviedo, 29-XI-1628, s/f (apud KaWAMURA, Y.: Arquitectura y poderes civiles..., p. 157). La figura de Horna aparece en los albores de la misma década cuando obtuvo el remate para enlosar la capilla de Nuestra Señora del Rey Casto de la catedral de Oviedo por la cantidad de 1.700 reales, teniéndose que acoger a las condiciones que había marcado el canónigo Diego Martínez de Galarreta (AHA, P0, caja 6934: Oviedo, 6-VII-1620, s/f).

16 Ramallo Asensio, G.: La arquitectura civil asturiana..., p. 104. y hazía de nuevo en las sus casas de la calle de San Juan» ${ }^{17}$. En la misma década de 1620 el obispo de Oviedo denunció a los Argüelles de Celles, regidores de la ciudad, y mandó embargar las obras de ampliación de la casa que estaban reformando en la corrada del Obispo por ocultación de luces sobre el palacio episcopal; a finales de la centuria los Llanes tendrán un conflicto similar con sus vecinos, los marqueses de Santa Cruz de Marcenado, propietarios del palacio de la Rúa, y la cofradía y hospital de la Balesquida, por la privación de luces sobre la propiedad hospitalaria y la apertura de una serie de vanos limítrofes con la propiedad de los marqueses, con los que el conflicto se alargaría hasta mediados del siglo XVIII ${ }^{18}$.

El hospital de San Juan el Real arguyó la ocultación de luces para denunciar la obra de don Diego de Miranda. La institución hospitalaria era la más antigua e importante de la ciudad, estaba bajo la administración del cabildo catedralicio y su finalidad era albergar los peregrinos que llegaban a la ciudad para la veneración de las reliquias de la Cámara Santa en su trayecto hacia Santiago de Compostela. $\mathrm{Su}$ fundación se vincula al antiguo edificio palaciego de Alfonso III, que fue donado por disposición testamentaria de Alfonso VI en 1096 al obispo y cabildo ${ }^{19}$. El ensombrecimiento de la calleja y el edificio de peregrinos pudo ser consecuencia del recrecimiento del palacio, lo que lleva a plantear el origen de la segunda planta del edificio.

El proceso constructivo de la reforma de Güemes es fragmentario y está vinculado al conflicto entre la Iglesia y el promotor. Como mencioné, el contrato con Horna para abastecer de piedra al maestro de la obra data del 29 de noviembre de 1628 y se alargaría por diez meses, pero por las actas capitulares del verano de 1628 se colige que la construcción estaba en un avanzado estado de gestación lo que dio la voz de alarma a los canónigos. En el capítulo del 17 de julio de 1628 se exponía los problemas

\footnotetext{
AHA, P0, caja 7045: Oviedo, 25-vIII-1629, s/f.

18 AMG, FM, cajas 55 y 58.

19 Sobre la iglesia, parroquia y hospital de San Juan vid. Risco, M.: España Sagrada. Tomo XXXVIIII. De la iglesia exenta de Oviedo. Desde mediado del siglo XIV a fines del siglo XVIII, Madrid, Oficina de la Viuda e Hijo de Marín, 1795; CANELLA y SeCADES, F.: El libro de Oviedo. Guía de la ciudad y su concejo, Oviedo, Impr. Vicente Brid, 1887; RuBIo BARDón, F.: Parroquia y templo de San Juan el Real de Oviedo, Oviedo, Parroquia de San Juan el Real, 1980; CABAL, M.: Hospitales antiguos de Oviedo, Oviedo, IDEA, 1985.
} 
de ventilación e iluminación que produciría la altura de los muros del palacio (fachada Norte) sobre la enfermería del hospital:

El señor doctor Menéndez, administrador del ospital de San Juan, pidió por una petizión que don Diego de Miranda alarga y alça la casa en mucho agravio del hospital; que nombren personas, lo bean y comuniquen con su Señoría y lo remedien. Nonbraron a los señores arcediano de Babia, chantre, canónigo Monterroso, doctor Naveda lo agan, y determinen lo que se aya de hacer. ${ }^{20}$

Para llegar a un consenso las partes nombraron comisarios que evaluaran los daños ocasionados, con la finalidad de evitar el pleito, que podía dilatarse en el tiempo y relentecería las obras, sin contar con el desembolso económico que ocasionaría; a estas circunstancias había que sumar el poder e influencia de la familia agravante, no solo en la ciudad, sino en la región, como ya había puesto de relieve en diversas ocasiones en el siglo anterior ${ }^{21}$. El propio mitrado dudaba de un resultado favorable en caso de juicio:

Los canónigos que el cabildo nonbró para ber el edificio que don Diego de Miranda hace en sus casas y si es de daño para la enfermería del hospital, respeto que con dicho edificio quita luz, sol y otras comodidades y que el ospital es fundación real y pía, dieron relación del agrabio ser grande y como lo an consultado con su Señoría que siente que en caso de duda de justicia y que don Diego repara el daño a satisfación de maestros; que se mire bien este negocio y no se enbarque el ospital en pleytos dudosos sin mucha justificación. Y el señor magistral, sin enbargo, como administrador contradijo el dicho edificio y lo pidió por testimonio, lo mismo hiço el señor arcediano de Tineo,

20 ACO, Libro de acuerdos capitulares, 1628-1636, tomo 23, capitulo del 17-vII-1628, fol. $438 \mathrm{v}$.

21 Los excesos de la familia Miranda ya habían quedado al descubierto en diversas ocasiones a lo largo del siglo xvi; ejemplos de ello pueden verse en FAYA Díaz, Ma ${ }^{\text {. }}$ Á.: Los señoríos eclesiásticos en la Asturias del siglo XVI, Oviedo, RIDEA, 1992 y «Los señoríos asturianos en tiempo de Felipe II", BIDEA, 158, 2001, pp. 109-121; Díaz Álvarez, J.: «Los marqueses de Valdecarzana, señores de vasallos en la Asturias del Antiguo Régimen (siglos xVI-XvIII)", Revista de Historia Moderna, 24, 2006, pp. 363-394. pidiendo entre anbos la causa se siga, que es bastante y justificada la causa y justicia que ay. Acordose por mayor, escepto los dichos referidos, en vista de la relación que el señor magistral y doctoral con Juan de Naveda y Gonzalo de Guemes, maestros, sin enbargo de los pareceres dados en conciencia, lo buelban a ber descargándose con la suya y con lo que el señor Obispo dijere, con que las ventanas se agan y juntamente solana en el distrito alto como conbenga, de suerte que los pobres queden con sol bastante; y en esta conformidad lo comuniquen con su Señoría y agan escrituras conforme más conbengan al ospital su decencia y autoridad sin que en nada en quanto a las curas y gocen de sol que deteriorada ni menoscavado; y todo a costa del dicho don Diego Miranda, encargándoles sus conciencias para que con ellas y con el parecer de su Señoría ellos cumplan con lo que deben. ${ }^{22}$ (El subrayado es mío).

Poco más de un año, el cabildo seguía quejándose de la fábrica de don Diego, insistiendo en la ocultación de luces como consecuencia de la altura que estaba ganando el edificio:

Acordaron sus mercedes que los señores arcediano de Ribadeo, y maestre escuela, y arcediano de Billaviciosa, doctor Menéndez, doctor Vara y doctor Naveda den cuenta al señor Provisor de lo tratado con el señor don Diego de Miranda sobre el hedificio de la casa que está labrando junto al ospital de San Juan, y dejar parar el daño que se hace al dicho ospital conforme a lo capitulado en presencia de su Señoría el señor Obispo; y que comuniquen los ynconbenientes y daños que se siguen al dicho ospital con el maestro Naveda para que se hexecute lo tratado y agan y ordenen este caso lo que más conbenga al bien del dicho hospital. ${ }^{23}$

Con la finalidad de llegar a un acuerdo de indemnización una comisión del cabildo se entrevistó con el noble. Fueron nombrados peritos cualificados: el maestro de obras catedralicio Juan de Naveda y su hermano el canónigo Francisco de Naveda, por parte de la Iglesia; y

\footnotetext{
22 ACO, Libro de acuerdos capitulares, 1628-1636, tomo 23, capítulo del 28-VII-1628, fol. 440 r.

23 Ibidem, capítulo del 14-VIII-1629, fol. $472 \mathrm{r}$.
} 
Gonzalo de Güemes y el regidor doctor Solares, por parte de Miranda. El acuerdo se concretó en el abono de tres mil reales al administrador de San Juan, el canónigo García de Arango: «con lo qual puede el dicho señor don Diego de Miranda proseguir y acavar su hedifızio como le paresziere" ${ }^{24}$.

Pero los problemas entre el aristócrata y el hospital no terminaron aquí. La estrechez de la calleja que separaba a las edificaciones se hacía más aguda por el entorpecimiento que provocaba un pilar del viejo edificio medieval, cuya eliminación y subsanación pretendía el cabildo corriera por cuenta de don Diego; además, una vez iniciados en la reparación se planteó la posibilidad de urbanizar los accesos a la iglesia parroquial de San Juan y su entorno, nivelando los suelos de la plazuela en la que confluían la calleja y la calle de San Juan (a la que estaba orientada la fachada occidental del palacio): "conforme el diseño de las casas del dicho señor Don Diego", empedrando este vial y habilitándole un sistema de drenaje de aguas. Por supuesto, el regidor se negó a tales sugerencias, que no llegaron a materializarse ${ }^{25}$.

Junto con la reforma del edificio residencial, el promotor encargó también la construcción de unas caballerizas, que hicieran las veces de cochera, pajar y granero. El abigarramiento del caserío del barrio de Socastiello, el más destacado de la ciudad por la proximidad de la fortaleza y cárcel del Principado, sede del poder de los corregidores en Asturias, impedía su erección anexo al palacio, por lo que hubo de emplearse un solar próximo, aledaño a la Plaza de la Fortaleza. La obra fue contratada con el maestro Martín de Posada, quien a su vez la traspasó a Pedro de Montesomo ${ }^{26}$. En un extracto de las rentas de la casa de Miranda $c$. 1645 se menciona este conjunto: «En la çiudad de Oviedo tiene casas principales y cavalleriças que nunca anduvieron en renta ${ }^{27}$. Esta segregación de funciones del mismo palacio, me inclina a suponer que la reforma del viejo solar de la calle de San Juan se dedicara integramente a lugar de habitación, por supuesto, con una diferenciación de usos en su interior, que distinguiera la zona de ocupación de la familia, de

AHA, P0, caja 7045: Oviedo, 25-VIII-1629, s/f. Ibidem.

AHA, P0, caja 7194: Oviedo, 27-vI-1631, s/f.

ARIDEA, Casa de Miranda, Arrendamientos: memorial de rentas, c. 1645 . la de la servidumbre y áreas de servicio; en ello insistiré más adelante. El establo fue adquirido por el segundo marqués de Camposagrado, don José Manuel Bernaldo de Quirós, en 1719 para la construcción de su palacio urbano, que ocupa toda la manzana: un edificio exento, realizado a partir de la casa de los Huergo en el lugar, vínculo que heredó el aristócrata de su madre. En la transacción, Camposagrado se comprometió a levantar un edificio de características semejantes, para tener los mismos usos en la calleja de San Juan ${ }^{28}$.

A finales de la década de 1660, los Miranda, ahora titulares del marquesado de Valdecarzana, encargaron una nueva reforma al recién llegado Gregorio de la Roza. ¿Qué necesidad pudo haber existido para invertir una nueva suma de dinero en el acondicionamiento del hogar aristocrático? Considero que hay que responder a este interrogante no desde lo material, sino desde lo cultural, desde el honor y la promoción social. La primera reforma coincide con la promoción de la familia al hábito de caballería de Santiago en la persona de don Diego de Miranda, que obtuvo en 1623; la segunda, aunque no exactamente en el tiempo, con la obtención del título de Castilla hacia 1642, en la persona de don Sancho de Miranda, hijo del anterior y padre del promotor de la segunda reforma; los servicios a la Corona a lo largo de la centuria iban en aumento y comienza a observarse una estrategia de asentamiento en la Corte; de hecho, el marqués don Lope fue el primero en la familia en obtener un oficio palatino: mayordomo de la reina Mariana de Austria, lo que pudo haber estado favorecido por su suegro, don Benito Trelles, presidente del consejo de Castilla, con cuya hija, doña Josefa Trelles Simó, marquesa de Torralba y de Bonnanaro, se casó en los años 1670. En todo caso, son dos épocas en las que la familia expresa su preeminencia en Oviedo.

Pocos son los datos que poseemos sobre el proceso constructivo de la reforma del maestro de la Roza. Lo que parece evidente es que su intervención pudo haber ocultado las novedades que hubiera podido aportar Güemes, más aún desde un punto de vista estilístico, pues se 
avanza desde un primer barroco sobrio, desnaturalizado, tendente al clasicismo post herreriano, al pleno, más naturalista y decorativista. Sendas reformas fueron destacadas, por la envergadura de las obras. En el contrato con de la Roza se especifica el acondicionamiento de «un cuarto de havitazión y para su disposición hicieron planta de la forma que se ha de fabricar ${ }^{29}$. Por la expresión, a priori, la magnitud de las obras no debiera parecer excesivo, pero considero necesario precisar el término "cuarto". No creo que se refiera a una de las salas del inmueble, sino a una porción importante del mismo: ¿acaso la cuarta parte? Esta suposición cobra sentido con una lectura atenta del contrato de obras entre el marqués don Lope y Gregorio de la Roza. Según el documento, el paño septentrional (orientado a la calleja de San Juan) había invadido 1,5 pies de la vía pública, lo que debía compensarse, retrocediendo la fachada principal la misma medida; esto obligó al arquitecto a recomponerla y ordenar los vanos de esta crujía, así como algunas de sus estancias ${ }^{30}$.

Estas causas pueden dar lugar a interpretar que la intrusión en el espacio público databa de la época de la reforma de Güemes, dando lugar al estrechamiento del mismo y la dificultad de paso, contribuyendo, junto a la elevación del edificio, a la ocultación de luces sobre el hospital. Pero como ya indiqué el conflicto con la institución eclesiástica tuvo lugar por la elevación del paramento, no por su movimiento hacia la vía pública, ¿puede proceder este problema de épocas anteriores?: la carencia de información al respecto impide una respuesta. La exigencia de compensar la intrusión con el retroceso de la fachada principal supone la destrucción de la contribución de Güemes en ella.

En la segunda mitad del siglo xvIII el vetusto palacio ovetense hacía tiempo que había dejado de ser habitado; los marqueses de Valdecarzana eran absentistas del Principado desde el segundo tercio de la centuria. En 1770 el canónigo don José Froilán de Heredia aforó por mil reales anuales el edificio para destinarlo como residencia de su sobrino, don Antonio de Heredia, titular de la Casa de Here-

\footnotetext{
29 AHA, P0, caja 7319: Oviedo, 4-v-1668, ff. 39r.-42r. (apud KaWAmuRA, Y.: Arquitectura y poderes civiles..., p. 158).

30 Ibidem.
}

$\mathrm{dia}^{31}$. El inmueble estaba lejos de ser un hogar confortable para los nuevos tiempos. Más raro resulta el hecho de que esta familia, integrante de la oligarquía ovetense desde la centuria anterior, carecía de casa propia; hasta su traslado vivían de alquiler en la calle de Cimadevilla, si bien una de las principales de la ciudad. Los contratos de foro, caracterizados por su larga duración, una o dos vidas, en ocasiones perpetuos, podían desfigurar la propiedad legal del edificio, por lo que Valdecarzana se aseguró su presencia en la ciudad, como linaje hegemónico y preeminente, exigiendo el mantenimiento de sus armas, sitas en las fachadas principal y oriental, y que el coste de las obras a corrieran por cuenta del forero ${ }^{32}$.

En una sociedad jurídicamente diferenciada como la del Antiguo Régimen, elementos honoríficos de ostentación y publicitación fueron esenciales, y uno de los ámbitos en los que se reveló: la heráldica. Con el contrato suscrito, los Heredia se verían privados de tal muestra, por este motivo su proyecto residencial se amplió. El solar aforado no estaba exento, lindaba al Sur con la casa de los Hevia, otra de las familias de la oligarquía de Oviedo desde el siglo XVI. Los Heredia adquirieron este segundo inmueble con el objeto de derruirlo y ampliar la vieja casa aforada, haciéndola exenta, tal como la conocemos en la actualidad. La nueva fachada Sur sería el elemento diferenciador de la nueva familia residente en la que poder exhibir sus armas; empero, no pasaría a ser la principal -como tal se mantuvo la oriental-, sino que se orientaba a un "xardinito capaz para el recreo de dicha casa como le tiene la del marqués de Camposagrado que se halla al frente de ellaw ${ }^{33}$. Esta circunstancia implicó la invasión de vía pública por lo que el canónigo propuso al ayuntamiento retroceder la fachada de los Hevia, igualándola con la de los Miranda, al sobresalir sobre ésta, de modo que se uniformizaría el paramento y la calle. Pero también hubo motivos más funcionales; la disposición

31 Sobre esta familia vid. MenÉndez González, A.: «Un patrimonio nobiliario: la Casa de Heredia (siglos XVI-XVIII)", BIDEA, 138, 1991, pp. 687-708.

32 González Santos, J.: «La casa del Campo: un palacio dieciochesco desconocido", Ástura. Nuevos Cartafueyos d'Asturies, 6, 1987, p. 24 y MAdrid Álvarez, V. de la: La arquitectura de la Ilustración..., p. 145.

33 AHA, P0, caja 8045: Oviedo, 29-VIII-1770, fol. $193 \mathrm{v}$. (apud Madrid Álvarez, V. de la: La arquitectura de la Ilustración..., p. 147). 
del terreno en desnivel, la mayor altura de la casa de los Hevia y el avance de su fachada orientada a la calle de San Juan fueron causa de ocultación de luces sobre la de los Miranda, lo que la hacía sombría y húmeda; en 1770 se describían las salas orientadas al muro Sur como "frías, melancólicas, oscuras y de mala disposizión y comodidad». La apertura de una fachada al mediodía solventaría estos problemas y dignificaría al conjunto residencial. El proyecto de reforma fue encargado a Manuel Reguera, si bien ejerció como maestro de obras poco tiempo. En 1771 el canónigo Heredia le interpuso un pleito, cuyos motivos se desconocen, y lo apartó de las obras. Éstas concluirían hacia $1774 . .^{34}$ Se obtuvo así un edificio cúbico, ordenado a través de un patio central, un viejo modelo consagrado dentro de la arquitectura residencial nobiliaria, tanto urbana como rural.

\section{Análisis estructural y decorativo}

La falta de documentación, así como el amplio número de reformas del edificio plantea diversas dudas razonables sobre la evolución del inmueble. Más que soluciones diseminaré hipótesis, que complementarán o intentarán descartar propuestas de otros investigadores. El aspecto actual del palacio responde al clásico esquema de cuatro crujías ordenadas alrededor de un patio central, que servirá como sistema de iluminación, de ventilación, de servicio y de distribución del espacio interno. La inclusión o no de torres que enmarcaran la fachada principal será, como adelanté, un tema de delicada interpretación. Según el profesor Ramallo Asensio el edificio contaría no con una torre como se observa en la actualidad, sino con dos, en su frente occidental ${ }^{35}$. La torre, como estructura constructiva vinculada a la residencia de la aristocracia no es una cuestión baladí, no deja de interpretarse como un modo más de dar lustre a la familia, atestiguar su antigüedad, la hidalguía de solar conocido del linaje al que se pertenezca, en total: la «encarnación suprema de la herencia y la tradición $»^{36}$. En todo caso,

34 Madrid Álvarez, V. de la: La arquitectura de la Ilustración..., pp. 142 y ss.

35 Ramallo Asensio, G.: Arquitectura civil asturiana..., p. 124.

36 SAltillo, Marqués del: "Palacios ovetenses. Datos para su historia (1476-1786)», Revista de la Universidad de Oviedo, IX-X, 1942, p. 267. no me muestro de acuerdo con Ramallo; sustento mi afırmación en algunas cuestiones, que si bien no son tajantes, pueden inducir a otra línea argumentativa válida.

En primer lugar, Juan Uría Ríu en su artículo no vincula ninguna edificación de estas características a los Miranda, documentación posterior de la casa tampoco hace alusión a torres, en cambio sí se referencia para los Quirós, como indiqué. Segundo, la falta del contrato de obras entre don Diego de Miranda y Güemes no permite asegurar con rotundidad que el primero incluyera la construcción de la torre hoy existente, ni tampoco en el contrato suscrito décadas más tarde con de la Roza; en cambio sí se desprende de la documentación, la elevación del edificio durante la primera reforma, que supone dar un piso más al conjunto, y la regularización de la fachada y compensar los metros de suelo público utilizados de más en 1628 con la retracción de la fachada principal en 1668. Un tercer motivo sería que una segunda torre en la esquina S-0, lindaría con la casa de los Hevia; quedaría desvirtuada, ensombrecida, camuflada por la casa de éstos, pues sobresalía por la de los Miranda, dado el desnivel de la calle de San Juan -ascendente en el sentido Norte-Sur-, como se indica en 1770: «La espresada casa de dicho don Joseph Lorenzo de Hevia lebanta sobre la de Miranda catorze pies de derecho, sin lo que lebanta de más el agres del texado que la cubre [...]»; además la vieja casa de los Hevia disponía de una distribución en altura diferente, repartida en cuatro pisos: planta baja, entresuelo, dos plantas y desván ${ }^{37}$. Una cuarta razón, más significativa que las anteriores me inclina a descartar la existencia de las dos torres durante las reformas barrocas; en un inventario de bienes de 1719 en el que se da cuenta de los objetos existentes en los palacios asturianos de los marqueses de Valdecarzana (los de Oviedo en la calle de San Juan y la llamada casa de Campo, el de la villa de Grado y el del coto de Villanueva en el mismo municipio, el del coto de Muros, y el de San Martín de la Plaza en Teverga), no se cita ninguna torre correspondiente al edificio que ocupa este trabajo; en cambio sí se mencionan en Grado y en Muros ${ }^{38}$. De este

${ }^{37}$ AHA, P0, caja 8045: Oviedo, 29-VIII-1770, ff. 192v. y ss. (apud Madrid Álvarez, V. de la: La arquitectura de la Ilustración..., pp. 150 y s.)

38 ARIDEA, Casa de Miranda, Inventario de los bienes muebles habidos en las casas que el marqués de Valdecarzana tiene en Asturias: la casa en la villa de Grado, 
modo, se anularía la hipótesis de las dos torres enmarcando una crujía principal. En todo caso, este elemento estructural no deja de ser difícil de explicar, si se tiene en cuenta el hecho de que, la torre conservada, parece de factura moderna.

En fin, de existir una segunda torre, la reforma de Reguera se hubiera visto forzada desmontarla, unificar alturas en la crujía occidental, como se hizo; y de volver a reedificarla, tendría que haberla dispuesto en la esquina S-0, en correspondencia con la del N-O. Pero, ¿hasta qué punto los Valdecarzana hubieran permitido demoler un elemento constructivo de tanta carga simbólica; más aún si tenemos en cuenta la prohibición de mudar sus escudos heráldicos de las fachadas occidental y oriental? En este orden de cosas, ¿pudo la torre existente ser un recrecimiento de la esquina $\mathrm{N}-\mathrm{O}$ llevado a cabo en algún momento del siglo xvIII, tras la citada fecha de 1719 y posterior a la reforma de Reguera, que no alude a este elemento constructivo en ningún momento? Téngase en cuenta que las décadas de 1710 y 1720 fueron de una cierta actividad constructiva por parte de los marqueses de Valdecarzana en Asturias: de la primera de las décadas data la última reforma y ampliación del palacio de Grado y la construcción de su magnífica capilla, bajo la advocación de Nuestra Señora de los Dolores y en Oviedo se llevó a cabo la construcción de una casa de recreo frente al Campo de San Francisco, llamada comúnmente la casa del Campo ${ }^{39}$. En todo caso, la torre conservada muestra una planta irregular, trapezoidal, lo que indica que no tiene un origen antiguo; a su configuración se llega por la mera superposición de pisos; un caso semejante se observa en el palacio avilesino de los marqueses de Ferrera, en el que se hace crecer una torre a mediados del siglo $\mathrm{XVII}^{40}$.

La falta de información impide tener una idea clara de la estructura del edificio antes de

la casa de Muros, la casa de Villanueva, la casa de Miranda y la casa del Campo en Oviedo. Año 1719.

39 González Santos, J.: "La casa del Campo...», pp. 23-32; Madrid Álvarez, V. de la: "Aportaciones documentales para el estudio del cuartel de milicias de Oviedon, Liño, 10, 1991, pp. 113-124 y "El palacio de Miranda-Valdecarzana y la capilla de Nuestra Señora de los Dolores de Grado", Liño, 11, 2005, pp. 103-125; Ruiz de LA PeÑA GonzÁlez, I.: La capilla de Nuestra Señora de los Dolores de Grado: construcción, historia y restauración, Salamanca, Asociación de Amigos de Grado, 2005.

40 Madrid Álvarez, V. de la: El palacio del marqués de Ferrera, Gijón, Trea, 2003, pp. 43 y ss. la reforma de Güemes. Ésta condicionó la construcción en el sentido de desproveerlo de una estructura clásica y jerárquica en la fachada en la que se diferenciara la planta baja o terrera, un entresuelo y el o pisos principales. Los dos primeros niveles se fusionan, de tal modo que se gana en espacio residencial y de servicio, dado que la función de caballeriza, cochera y granero se concentraba en un edificio independiente. En la planta baja se abren vanos cuadrangulares, quizás para recibir el mayor aporte lumínico posible, seguidos por otros de hechura abalconada; este nivel bajo equivale a un entresuelo. En planta, las crujías se ordenan alrededor de un patio central, el conservado de la segunda mitad del siglo xviII debido al proyecto de Reguera. El doble piso resulta novedoso dentro de la estructura urbana residencial; lo más habitual fue habilitar un solo piano nobile, este procedimiento ha de vincularse a la primera reforma barroca (1628); Reguera se vio condicionado y la mantiene.

Las fachadas Oeste, Este y más tarde la meridional son íntegramente de sillería, y los niveles se ordenan a través de líneas de imposta. Tan solo queda discordante la septentrional, remodelada en la década de 1970 durante la rehabilitación del edificio, siendo sustituido el paramento por una estructura lisa enlucida con diversos huecos de iluminación en disposición regular. Cabe considerar que el muro original, careciera de un valor plástico apreciable para su conservación y no parece que hubiera motivo para suponer su factura también en sillería. Impostas y esquinales sobresalen del plano de los muros. Los vanos están dispuestos de un modo jerárquico; los del piso bajo son cuadrangulares y equivaldrían a salas de servicio; en el piso noble se abren balcones volados que apoyan en la imposta, en el piso superior la balconada es más sencilla y se abre directamente sobre el muro.

El actual patio porticado alrededor del que se organizan las crujías se debe al proyecto de Reguera; no queda rastro del modelo que pudo haber propuesto de la Roza, y menos aún del de Güemes. Se caracteriza por una triple arcada (arcos carpaneles) en cada uno de los lados, que apoya en una columnata de orden toscano y fustes lisos. En la planta baja, el espacio porticado está dividido en cada sección de arco con fajones carpaneles y cajeados, que parten de las columnas hasta las ménsulas molduradas 
del muro opuesto, utilizándose como cubierta bóvedas de arista ${ }^{41}$.

La intervención de 1668 afectó sobre todo al ala occidental, la principal, la orientada a la calle de San Juan. Dado que el marqués don Lope tuvo que retroceder la fachada, su nueva fábrica se exigió en cantería, dotando de molduras a los vanos que se practicaran: "mazizar de sillería, labrándola con la misma hermosura que puertas y ventanas" ${ }^{42}$. El acceso al edificio se hace a través una portada adintelada que se abre a un zaguán, que da paso al patio. La suficiente altura de la planta baja permitió a de la Roza un entresuelo, no tan visible en el exterior, por el desnivel ascendente de la calle. Empero, una de las características de la fachada principal es su disposición asimétrica. Se distingue en el extremo N-O la torre, enmarcada por esquinal y muro medianero, en la que los vanos que se practican en su lados N y 0 marcan un claro eje de simetría. En el resto del muro se practican otras cinco aberturas: cuatro ventanas cuadrangulares y la portada en la planta baja, alineados con los cinco balcones de los pisos superiores. Los tres primeros se distribuyen de un modo regular, como si hubiera una correspondencia con otro espacio gemelo a la primera torre, y en su lugar se practican dos huecos que marcan la asimetría. Una medianera separa la casa de los Miranda de la ampliación de los Heredia, que mantiene el mismo esquema compositivo. La asimetría de esta fachada se observa también en otra obra de Roza, en el cercano palacio de los Malleza. ¿Puede esta articulación subordinarse a la disposición del patio interior? En época de las reformas barrocas, el solar de los Miranda era menor, lo que obligaría a un posible patio oblongo; en el caso del palacio de Malleza, el patio parece alinearse con la portada y los dos vanos que la flanquean; el boceto de las trazas de este edificio data de 1669, si bien las obras se inician un año más $\operatorname{tarde}^{43}$. En el caso que me ocupa, el contrato suscrito entre de la Roza y el marqués don Lope establece un esquema bien calculado: proporciones en el grosor de los muros, en las medidas de las ventanas, en el ancho de las líneas de imposta, en la altura de cada uno de los pisos, que decrece según se gana en altura con

\footnotetext{
${ }^{41}$ MAdrid Álvarez, V. DE LA: La arquitectura de la Ilustración..., p. 151.

42 AHA, PO, caja 7319: Oviedo, 4-v-1668 (apud KaWAMURA, Y.: Arquitectura y poderes civiles..., p. 158).

43 KaWAmura, Y.: «Precisiones sobre la construcción...», p. 163.
}

la finalidad de acentuar el piso noble. En este esquema se solicita con toda claridad el desplazamiento de la portada hacia la parte baja de la calle, lo que no obscurece el sentido ordenador del espacio, a lo que contribuyen los dos vanos que la flanquean: «sin antepecho, del mismo claro en alto y ancho [...] dejándolas en proporzión que los dinteles vengan [...] con el de la puerta principal, acomodándolas en la distancia que ay de zaguán»" ${ }^{44}$.

La crujía con decoración más sobria es la occidental. Los vanos del piso inferior están ligeramente enmarcados por sillares; tan sólo destaca la portada, flanqueada por sendas pilastras cajeadas y partidas. Dado que la primera reforma del edificio data de los últimos años de la década de 1620 si este elemento ornamental fuera propuesto por Güemes resultaría un claro antecedente para la cercana torre monástica de San Pelayo el Real planteada por Melchor de Velasco mediada la centuria $^{45}$; la modificación de la fachada por de la Roza desmontará este supuesto como expondré más adelante. La portada está recorrida por unas ligeras molduras; sobre ella un friso continuo alberga cuatro rosetas ordenadas dos a dos en los espacios que permiten tres ménsulas -una en el eje longitudinal de la portada-, y que sirven como elemento sustentante de la línea de imposta y el volado del balcón corrido superior. La sobriedad ornamental de la fachada se ameniza, en algún grado, por la decoración heráldica, elemento imprescindible de ostentación para el grupo social nobiliario, que se dispone en el eje longitudinal alineado con la portada. Esta sobriedad compositiva y decorativa puede inducir a pensar que fuera diseño de Güemes, aunque las circunstancias que rodean la practicada por de la Roza pueden inducir a descartar este supuesto, o no, dado que en el contrato de 1668 se pide una ornamentación con motivos vegetales: "y todo el follage que en [la] arquitectura quepa y se pudiere, sin quitar nada del bueco y [la] puertan ${ }^{46}$. Puede que esta petición se hubiera reducido a las cuatro rosetas y

44 AHA, PO, caja 7319: Oviedo, 4-v-1668 (apud KAWAMURA, Y.: Arquitectura y poderes civiles..., 158).

45 Ramallo Asensio, G.: La arquitectura civil..., p. 124 y «El arquitecto Melchor de Velasco antes de su llegada a Galicia", en Tiempo y espacio en el arte. Homenaje al profesor Antonio Bonet Correa, Madrid, Universidad Complutense, 1994, pp. 501-523; Madrid Álvarez, V. de la y G. Ramallo AsENSIO: "Arquitectura barroca religiosa (II)», en $\mathrm{El}$ arte en Asturias a través de sus obras, dir. J. Barón Thaidigsman, Oviedo, Prensa Asturiana, 1996, p. 247.

46 AHA, PO, caja 7319: Oviedo, 4-v-1668 (apud KaWAMURA, Y.: Arquitectura y poderes civiles..., p. 158). 
ménsulas naturalistas que decoran el friso de la portada. Cuesta pensar en una continencia ornamental tan llamativa bien avanzado el siglo. Este hecho viene a reforzar la hipótesis de un barroco ambivalente, extremadamente contenido en la arquitectura tanto urbana como rural, junto a otro más expresivo, grandilocuente, jubiloso. Este doble sentido es más elocuente si tenemos en cuenta la intervención de Roza en el cercano palacio de Malleza -también muy próximo en el tiempo-, cuya portada es mucho más decorativa y en la que se refleja un mayor número de referencias ornamentales de tendencia naturalista. En todo caso, la axialidad de la fachada principal de la casa Valdecarzana está regida por la simetría.

La intervención de Manuel Reguera, para los Heredia, en la segunda mitad del siglo XVIII destaca en el lienzo Sur. Dada su orientación al jardín y que éste estaba tapiado por un muro de mampostería, el emblema heráldico debía ser bien visible por lo que se dispone en el segundo piso, en el eje de simetría de la fachada, visible sobre la tapia. Esta fachada se organiza en tres niveles: bajo y dos alturas, divididos por líneas de imposta y su factura es integramente de cantería bien labrada y escuadrada. Las ventanas del piso bajo son cuadrangulares y están enmarcadas por molduras lisas, a excepción del hueco central en el que se practica una puerta de acceso al jardín.

Dada la pronta desvinculación de Reguera del proyecto del canónigo Heredia y su sustitución por José Bernardo de la Meana, la intervención del primero, en esta fachada es muy posible que se redujera al aspecto compositivo y ordenador de los vanos. La cuestión estética y decorativa quedaría sujeta al segundo. Los vanos de los pisos superiores son abalconados. Los del principal son volados y están rematados por arcos muy rebajados; los del superior adintelados y sin volar. Los primeros concentran la mayor riqueza decorativa al estar rematados por dos soluciones alternas: dos volutas unidas por una venera y una moldura mixtilínea en cuyo eje central incorpora una máscara esculpida. Esta última composición recuerda a la que proporcionó Pedro Antonio Menéndez en el vecino palacio de Camposagrado y a la fachada del Hospicio, en la que también trabajaría de la Meana. En la ampliación de los Heredia la fachada dispone siete calles, la central más monumental, marcando el eje axial desde la portada al balcón principal sobre el que se dispone el escudo de armas de los promotores. La primera rompe la línea de ventanas cuadrangulares, se remata con un arco rebajado y está flanqueada por sendas pilastras cajeadas y almohadilladas; el balcón de honor, volado, está entre dos columnas toscanas estriadas, que sustentan un entablamento quebrado de triglifos y metopas que custodia motivos naturalistas; sobre todo este conjunto pesan las armas de la familia, que rompe la balconada del piso superior, de nuevo dispuesto por el mismo esquema de pilastras de la portada, cerrando el ciclo ${ }^{47}$.

Los motivos que mueven a crear esta fachada meridional son precisamente los que la hacen singular. No se trata de una fachada más. Reguera debe conjugar dos supuestos, por un lado simular su importancia, como si fuera la principal, orientada a la calle pública; de otra parte, ha de concebirse como un divertimento, como un marco idóneo para el espacio natural al que se orienta, el jardín, elemento de descanso y esparcimiento de la familia. Podría decirse que el precedente inmediato de este contexto es el vecino palacio de Camposagrado, pero el acceso al jardín no se tuvo en cuenta desde un principio, pues se vio en la obligación de practicar un hueco de acceso en el muro Sur rompiendo la composición del basamento y entresuelo. En Heredia es diferente, el diseño se tiene en cuenta desde el principio influyendo en el diseño final. Es muy posible que pesara la estancia de Reguera en la Corte para obtener su título de arquitecto. Carlos III deseoso de acomodar la Corte a las necesidades y requerimientos de una gran urbe europea, favoreció reformas urbanísticas, lo que coincide también con la adaptación a los nuevos conceptos de habitabilidad y de domesticidad de las viejas residencias. En este contexto, no fue raro demandar un espacio naturalista privado, un jardín, y emplazar una de las fachadas hacia él, si bien en los casos de nuevas construcciones, hubo casos de exigir que el complejo constructivo se incluyera en una amplia cinca ajardinada urbana, al modo francés, frente a la moda castellana de emplazar el edificio a pie de calle ${ }^{48}$; el

\footnotetext{
47 Madrid Álvarez, V. DE LA: «La arquitectura palaciega de Pedro Antonio Menéndez y Manuel Reguera", en Arquitectura señorial en el norte de España, dir. G. Ramallo Asensio, Oviedo, Universidad de Oviedo, 1993, p. 126; La arquitectura de la Ilustración..., pp. 152 y ss.; "Arquitectura de la Ilustración (I)", en El arte en Asturias..., pp. 272 y ss.

48 Sobre estos asuntos vid. López-Cordón Cortezo, M. ${ }^{a}$ V.: "Casas para administrar, casas para deslumbrar: la pedagogía del palacio en la España del siglo xviII", en El mundo urbano en el siglo de la Ilustración, eds. 0. Rey
} 
mejor ejemplo es el palacio demandado por don Jacobo Fitz-James Stuart Ventura Colón, tercer duque de Berwich y de Liria, a Ventura Rodríguez en 1773. En Oviedo, el palacio de Miranda-Heredia se adapta a esa nueva necesidad doméstica, que denotaba exclusividad, más aún en un reducido espacio urbano como el ovetense, donde la escasez de solares intramuros era una constante; su finalidad fue la de propiciar distracción al individuo y su recreo en la naturaleza desde un punto de vista privado; además de ser reflejo del poder económico y preeminencia social. Sobre el espacio ajardinado privado ya habían tratado los círculos ilustrados. El aristócrata vasco don Francisco Xavier María de Munibe e Idiáquez, octavo conde de Peñaflorida y cofundador de la Sociedad Vascongada de Amigos del País, teorizó sobre él cuando escribió:

El jardín en una ciudad es un gran recurso: la ventilación que facilita al edificio, la bella vista, las flores, las frutas y las ensaladas lo constituyen precioso. Tiene uno en su casa el paseo, que lo encuentra a todas las horas sin haberlo de ir a buscar fuera: va medio vestir, no ve sino lo que quiere ver y se libra de importunos. Si el terreno es capaz, se debe tener patio y jardín a un tiempo, observando cuanto sea posible situar el jardín de modo que las vistas de los vecinos no den a él. ${ }^{49}$

\section{Conclusiones}

La casa de Miranda, titular del marquesado de Valdecarzana, fue una de las más preeminentes de Asturias, lo que se sustentó en antiguos derechos bajomedievales, ser cabeza de bando y de parcialidades hasta bien entrado el siglo xvI, y parientes mayores del Principado. Bajo estas premisas, sus titulares dispusieron de

Castelao y R. J. López, t. II, Santiago de Compostela, Xunta de Galicia, 2009, pp. 17-53; GonzÁlez Heras, N.: «De casas principales a palacio. La adaptación de la residencia madrileña a una nueva cotidianeidad", Revista de Historia Moderna, 30, 2012, pp. 47-66.

49 Peñaflorida, conde de: "Discurso sobre la comodidad de las casas que procede de su distribución exterior e interior", en Ensayo de la Sociedad Bascongada de los Amigos del País. Año de 1766, Vitoria, Impr. Thomás de Robles, 1768, pp. 233 y s. Sobre este "Discurso" vid. León Tello, F. J. y M. ${ }^{a}$ V. SAnz SAnz: Teoría de la arquitectura en los tratados españoles del siglo xvIII, Madrid, CSIC, 1994, pp. 240 y ss. residencia en Oviedo, principal centro urbano y foro político asturiano, en un lugar privilegiado: la calle de San Juan, en el barrio de Socastiello, donde se levantaba la fortaleza real, sede del corregimiento. El viejo edificio documentado desde la primera mitad del siglo xvI $\mathrm{y}$ de unas trazas medievalizantes tuvo que ser adaptado a los nuevos usos domésticos, de habitabilidad y de civilidad que se estaban asentando en la sociedad; pero al mismo tiempo, el ascenso social y los honores recibidos de la Corona, como fue hábito santiaguista en 1623 y el título de Castilla en 1642, debió de exteriorizarse al resto de los convecinos, publicitando el linaje con expresiones como la heráldica y la magnificencia del hogar; de este modo se explican las reformas barrocas de 1628 y de 1668 a cargo de dos maestros destacados y vinculados a la vanguardia constructiva, Gonzalo de Güemes y Gregorio de la Roza, respectivamente, relacionados con las estéticas post herrerianas en la que destacó también Juan de Naveda y el barroco pleno a través del magisterio de Ignacio del Cajigal.

El absentismo de los Valdecarzana desde el segundo tercio del siglo xviII supuso el abandono del vetusto caserío y favoreció su aforamiento a otra familia de la oligarquía urbana, los Heredia. El edificio tuvo que acondicionarse de nuevo a los nuevos modos de vida del siglo "que llaman ilustrado». En esta ocasión se contó con la colaboración del primer arquitecto titulado por la Real Academia de Bellas Artes de San Fernando, Manuel Reguera, y de José Bernardo de la Meana. La exigencia de los marqueses propietarios de mantener la estructura del edificio y sus armas en lugar de honor, hizo que los nuevos inquilinos adquirieran el solar colindante, lo derribaran y adaptaran una nueva traza que se fusionara con la casa de los Miranda: el resultado fue un edificio cúbico, exento, y la nueva ala, la meridional, de un nuevo gusto, si bien no neoclásico, tendente a la sobriedad. Una de las finalidades era disponer de una fachada propia en la que exhibir su escudo heráldico. La influencia del vecino palacio Camposagrado en las molduras decorativas de los balcones, a cargo de la Meana y la estancia de Reguera en la Corte en 1764 se dejan sentir en la ordenación de la nueva fachada orientada a un jardín privado; una fachada orientada al mediodía, más abierta, que imprimirá mayor luminosidad a sus salas. 
